\title{
Three-dimensional self-similar fractal light in canonical resonators
}

Steven W. Miller, John Nelson, Johannes Courtial

Steven W. Miller, John Nelson, Johannes Courtial, "Three-dimensional selfsimilar fractal light in canonical resonators," Proc. SPIE 9194, Laser Beam Shaping XV, $91940 Z$ (2 October 2014); doi: 10.1117/12.2061410

EDIE Event: SPIE Optical Engineering + Applications, 2014, San Diego, California, United States 


\title{
Three-dimensional, self-similar, fractal light in canonical resonators
}

\author{
Steven W. Miller, John Nelson, and Johannes Courtial \\ School of Physics \& Astronomy, University of Glasgow, Glasgow G12 8QQ, United Kingdom
}

\begin{abstract}
Unstable canonical resonators can possess eigenmodes with a fractal intensity structure [Karman et al., Nature 402, 138 (1999)]. In one particular transverse plane, the intensity is not merely statistically fractal, but selfsimilar [Courtial and Padgett, PRL 85, 5320 (2000)]. This can be explained using a combination of diffraction and imaging with magnification greater than one: each round trip through the resonator adds approximately the same diffraction pattern to the resonator, which gets then magnified through imaging, resulting after many round trips in the diffraction pattern being present on a cascade of length scales, a hallmark of fractals. Here we show that the same mechanism also shapes the intensity cross-section in the longitudinal direction. Combined with the mechanism for shaping the transverse intensity distribution, this results in three-dimensional, self-similar, fractal intensity structure in the lowest-loss eigenmode. Because the transverse and longitudinal magnifications are different, the scaling properties in the transverse and longitudinal directions are different. We demonstrate this structure using computer simulations.
\end{abstract}

Keywords: fractal laser modes, unstable canonical resonators, scalar wave optics, self-similarity

\section{INTRODUCTION}

Fractal geometry is arguably the geometry of nature: ${ }^{1}$ the shape of trees, grasses, clouds and mountains can all be described by fractal geometry. Fractal patterns are characterised by self-similarity: the pattern consists of parts which are similar to the whole, either exactly (like in famous fractals such as the Sierpinski gasket, which consists of three half-size Sierpinski gaskets) or statistically (like in certain clouds). The range of length scales over which this self-similarity applies, the scaling range, is limited in physical fractals, in light distributions usually by diffraction ${ }^{2,3}$ Fractal light distributions can result from interaction with fractal objects, ${ }^{4-7}$ from random processes $^{8}$ and from diffraction upon propagation after passage through a Ronchi grating ${ }^{9}$ - the fractal Talbot effect.

Successive round trips through optical resonators can also shape light beams into fractals. This was noticed for optical resonators specifically designed to possess fractal eigenmodes ${ }^{10-12}$ (and for the incoherent versions of such resonators ${ }^{13,14}$ ). At the same time it was independently realised that the technologically important class of unstable canonical optical resonators - two spherical mirrors facing each other - also possess an innate tendency to shape light into fractals. ${ }^{15,16}$ This latter discovery caused significant activity, with various groups studying the fractal properties of the eigenmodes of unstable canonical optical resonators. ${ }^{15-21}$ Berry and coworkers highlighted a link between the Ronchi grating and unstable canonical optical resonators: light from different unit cells in the former corresponds to light from different round trips in the latter. ${ }^{18}$

The mechanism by which unstable canonical optical resonators shape light into fractals is particularly clear in a particular plane, the magnified self-conjugate plane, which one round trip through the resonator images back into itself with a magnification with a modulus greater than one. ${ }^{22,23}$ The mechanism for the generation of fractal structure is a combination of repeated imaging and diffraction. The former occurs also in video feedback, where pixellation can play the role of diffraction, and the combination can result in fractal patterns ${ }^{24,25}$ (Fig. 1). (The ray-optical nature of the repeated imaging in this process was also highlighted by ray-tracing simulations of the view inside canonical optical resonators. ${ }^{26}$ ) Because of this analogy with video feedback, the mechanism

Further author information: JC's e-mail address is johannes.courtial@glasgow.ac.uk

Laser Beam Shaping XV, edited by Andrew Forbes, Todd E. Lizotte, Proc. of SPIE Vol. 9194, 91940Z ( 2014 SPIE · CCC code: 0277-786X/14/\$18 - doi: 10.1117/12.2061410 
(a)

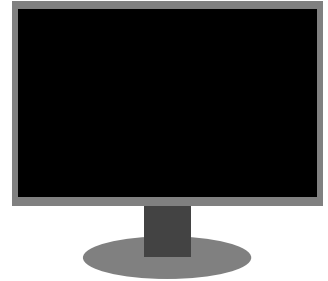

$n=0$

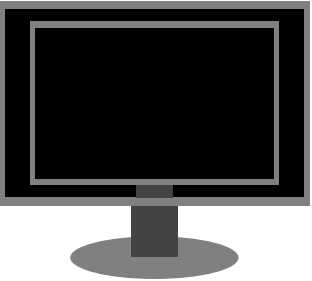

$n=1$

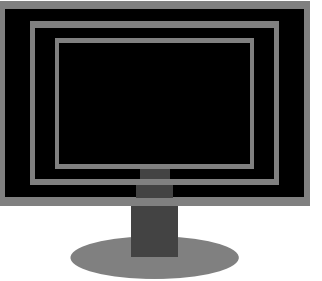

$n=2$

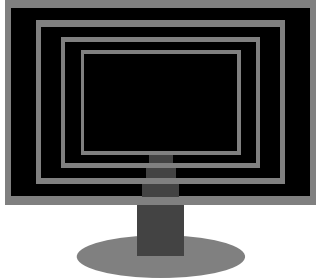

$n=3$

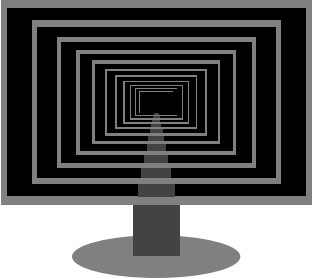

$n=10$

(b)
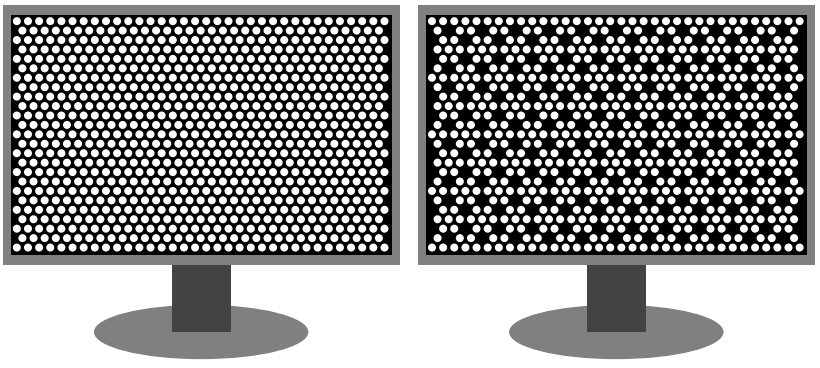

$n=0$

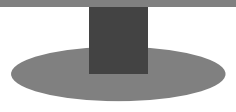

$n=1$
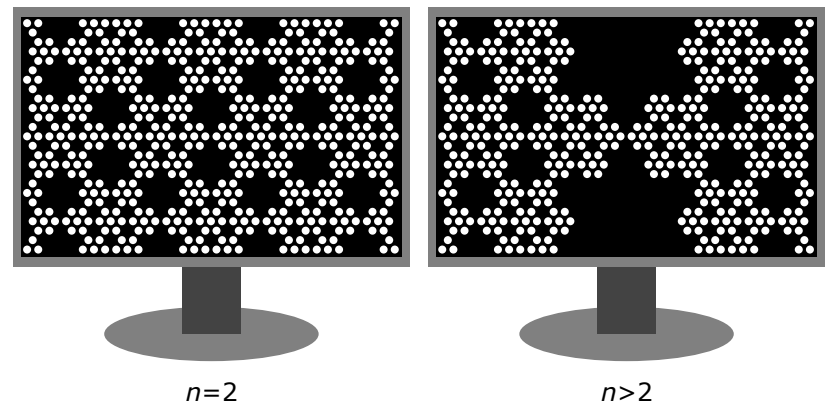

Figure 1. Monitor-inside-a-monitor effect, also known as Droste effect, and monitor-outside-a-monitor effect. A video camera pointed at a monitor displaying the image it is recording results in video feedback. In general, the image is displayed with a size different to its original size; it is scaled by the video-feedback magnification. (a) If the image is de-magnified, i.e. if the video-feedback magnification is $<1$, the result is the monitor-inside-a-monitor effect. Here the camera records an image of the monitor, and displays this image, with magnification $M=0.8$, on the monitor. Initially, after $n=0$ video-feedback loops, the monitor is blank. After $n=1$ loop, the monitor shows a reduced-size image of itself. After $n=2$ loops, it shows a reduced-size image of itself showing a reduced-size image of itself. Generally, after $n$ loops the monitor shows $n$ images of itself at magnification $M, M^{2}, M^{3}, \ldots, M^{n}$. (b) When the video-feedback magnification, $M$, is $>1$, and if the monitor has pixels, the monitor-outside-a-monitor effect occurs. In the example shown here, the monitor initially displays a white image, i.e. all pixels are white. The camera records the pixel pattern in the central area of the screen, and after $n=1$ video-feedback loop, this centre of the pixel pattern is displayed on the monitor, a factor $M=3$ larger. But because the enlarged pixel pattern is displayed on the monitor, which is pixellated, the pixel pattern is then present in two sizes: the original size, and enlarged by a factor $M$. After the next feedback loop, the pixel pattern is present in three sizes, namely enlarged by factors $1, M$, and $M^{2}$, and each subsequent feedback loop adds the pattern at a larger magnification. This presence of a pattern on a cascade of length scales is a hallmark of fractals. 


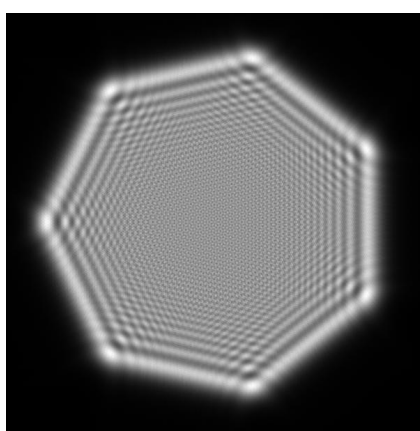

$n=1$

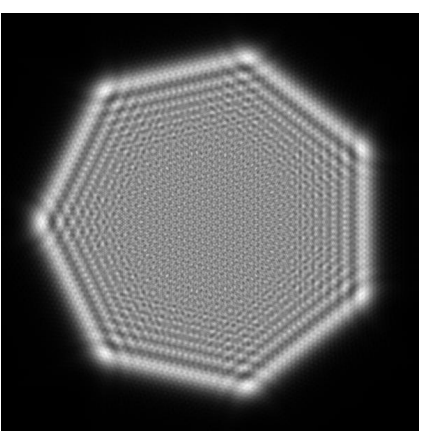

$n=2$

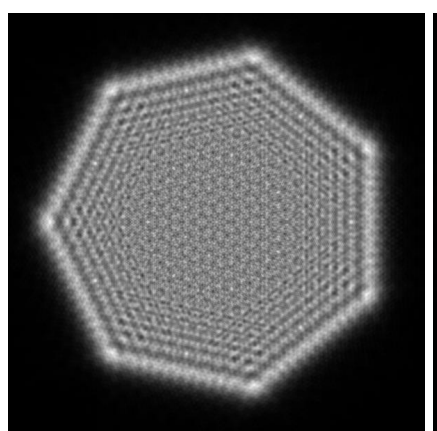

$n=3$

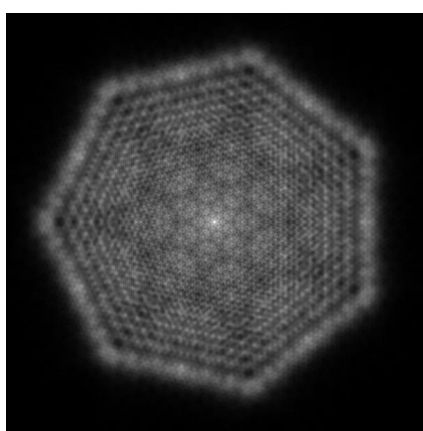

$n=20$

Figure 2. Intensity cross-section in the magnified self-conjugate plane of an unstable resonator with a Koch snowflake eigenmode ${ }^{23}$ after $n$ round trips through the resonator, starting with a uniform plane wave. The simulation was performed using our custom wave-optics simulator Young TIM. ${ }^{27}$

for this generation of fractal patterns was called the monitor-outside-a-monitor effect. Remarkably, the monitoroutside-a-monitor effect produces light distributions that are, within the diffraction limit, exactly self-similar, not merely statistically self-similar like the light distributions in other transverse planes.

Here we point out that the ingredients of the monitor-outside-a-monitor effect in unstable canonical resonators are present not only in the magnified self-conjugate plane, but also on the optical axis and even in the volume surrounding the point where the optical axis intersects the magnified self-conjugate plane. We present simulations that demonstrate the generation of fractal eigenmode distributions.

\section{MONITOR-OUTSIDE-A-MONITOR EFFECT}

The way in which the monitor-outside-a-monitor effect creates fractals in video feedback is explained in Fig. 1. Starting with uniform white displayed on the pixellated monitor, the camera records the centre of the pixel pattern and displays it, magnified by a factor $M$, on the pixellated monitor, which imprints the pixel pattern at its original size onto the displayed image. Each subsequent video-feedback loop magnifies all pixel patterns present by a factor $M$ and adds another original-size pixel pattern. In this way, after $n$ loops the pixel pattern is present at magnifications $1, M, M^{2}, M^{3}, \ldots, M^{n-1}, M^{n}$.

In unstable canonical optical resonators, the diffraction pattern due to any apertures present in the resonator plays the role of the pixel pattern. In the laser, this pattern gets added to the enlarged pattern from the previous round trip, whereas in video-feedback it gets imprinted upon the enlarged pattern from the previous feedback loop. Geometrical imaging of the magnified self-conjugate plane onto itself plays the role of recording the image on the monitor by the camera and displaying it again on the monitor. Details can be found in Ref. ${ }^{22}$ Fig. 2 shows an example of the intensity distribution in the magnified self-conjugate plane of a canonical resonator containing a 7 -sided polygonal aperture after $n=1,2,3$ and 20 round trips, at which point it has settled into a very close approximation to the lowest-loss eigenmode. The initial pattern is a uniform plane wave.

But the two ingredients of the monitor-outside-a-monitor effect in lasers occur not only in the magnified self-conjugate plane: the diffraction pattern due to the apertures in the resonator exists not only in the selfconjugate plane, but in the entire volume of the resonator, and the resonator's mirrors image not only the magnified self-conjugate plane, but again the resonator's entire volume. It is therefore reasonable to search for diffraction-limited but otherwise exactly self-similar 3D fractal intensity structure in the eigenmodes of unstable canonical optical resonators.

One complication is that imaging by lenses and mirrors is such that the magnification in the longitudinal direction, $M_{L}$, is the square of the magnification in the transverse directions, $M$. Unless both these magnifications are 1 they are therefore different from each other. This means that the self-similarity of the intensity patterns resulting from the 3D monitor-outside-a-monitor effect is such that the patterns must be stretched by a factor $M_{L}$ in the longitudinal direction and by a (different) factor $M$ in the transverse directions before they look similar 


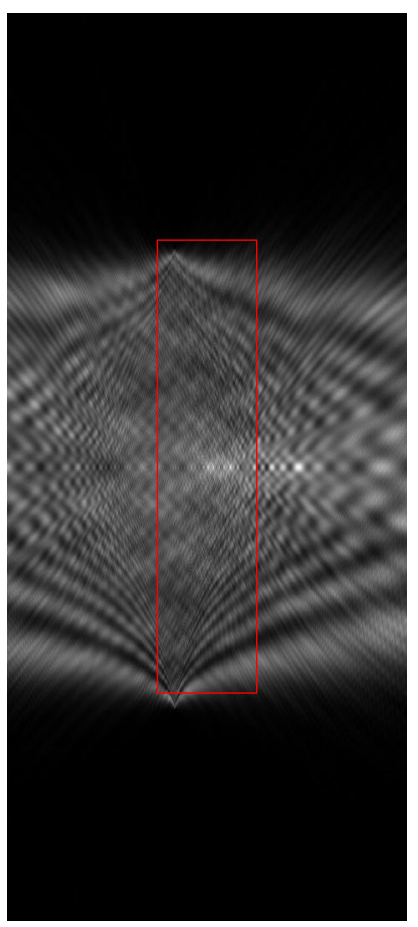

$x 1$

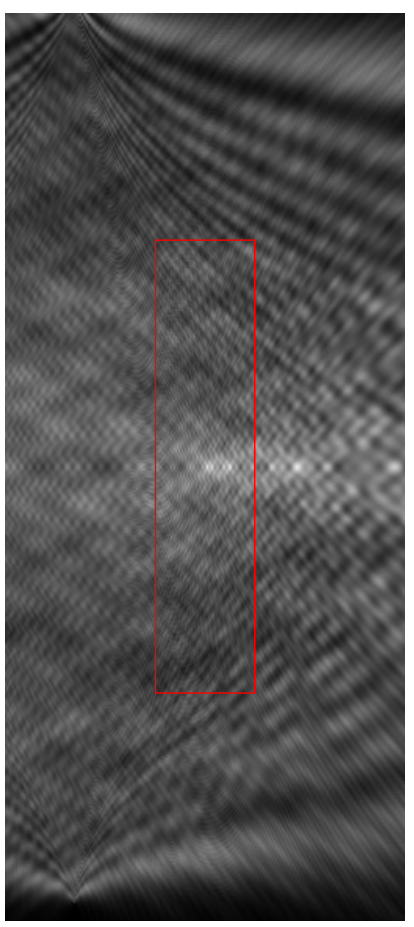

$x(4,-2)$

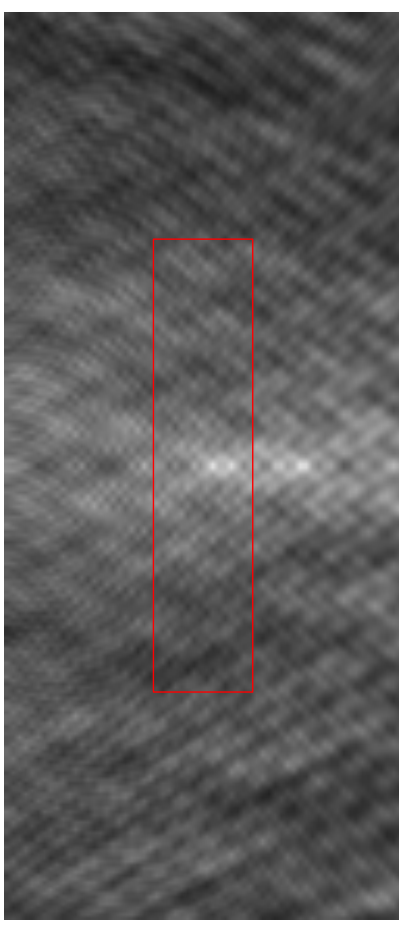

$x(16,4)$

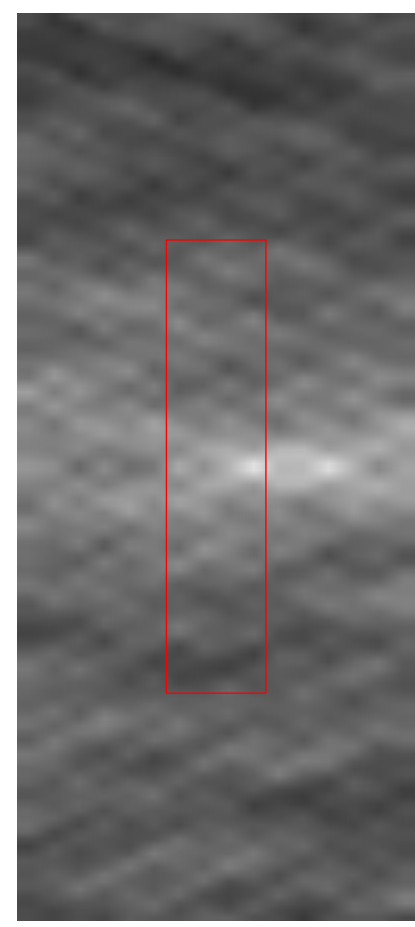

$x(64,-8)$

Figure 3. Self-similarity of the lateral intensity cross-section of the beam after $n=10$ round trips, starting with a uniform plane wave, through the same resonator for which Fig. 2 was calculated. The longitudinal direction in the resonator is shown in the horizontal direction, the (transverse) horizontal direction in Fig. 2 is shown in the vertical direction. The different frames show the centre of the intensity distribution, successively magnified by a factor $M=-2$ in the vertical direction and by $M^{2}=4$ in the horizontal direction; the overall magnifications in the horizontal and vertical directions by which each frame has been magnified relative to the left frame is listed below each frame. The red frame shows the part of the cross-section shown in the next frame. All frames are centred horizontally on the magnified self-conjugate plane and vertically on the optical axis. The simulation was performed in our custom wave-optics simulator Young TIM. ${ }^{27}$ 
again to the unstretched pattern. Fig. 3 demonstrates this effect for the beam after $n=10$ round trips (which is almost identical to the beam after 20 round trips) for the same resonator for which Fig. 2 was calculated.

\section{CONCLUSIONS}

We have found three-dimensional, diffraction-limited exactly self-similar fractal intensity structure in the lowestloss eigenmodes of unstable canonical optical resonators. We explain this structure in terms of a 3D monitoroutside-a-monitor effect: the interplay between the 3D imaging properties of unstable resonators and diffraction. Due to the characteristics of geometrical imaging with curved mirrors, the self-similarity of this intensity structure is such that it must be stretched by different factors in the transverse and longitudinal directions to be similar to itself again.

\section{REFERENCES}

[1] B. B. Mandelbrot, The Fractal Geometry of Nature, Freeman, San Francisco, New York, 1982.

[2] D. Avnir, O. Biham, D. Lidar, and O. Malcai, "Is the geometry of nature fractal?," Science 279, pp. 39-40, 1998.

[3] B. B. Mandelbrot, "Is nature fractal?," Science 279, pp. 783-784, 1998.

[4] M. Lehman, "Fractal diffraction gratings built through rectangular domains," Opt. Commun. 195, pp. 1126, 2001.

[5] G. Saavedra, W. D. Furlan, and J. A. Monsoriu, "Fractal zone plates," Opt. Lett. 28, pp. 971-973, 2003.

[6] D. Werner and S. Ganguly, "An overview of fractal antenna engineering research," Antennas and Propagation Magazine, IEEE 45, pp. 38-57, 2003.

[7] M. W. Takeda, S. Kirihara, Y. Miyamoto, K. Sakoda, and K. Honda, "Localization of electromagnetic waves in three-dimensional fractal cavities," Phys. Rev. Lett. 92(9), p. 093902, 2004.

[8] K. O'Holleran, M. R. Dennis, F. Flossmann, and M. J. Padgett, "Fractality of light's darkness," Phys. Rev. Lett. 100(5), pp. 053902-4, 2008.

[9] M. V. Berry and S. Klein, "Integer, fractional and fractal Talbot effects," J. Mod. Opt. 43, pp. 2139-2164, 1996.

[10] J. Courtial, "Fractal resonator modes." CLEO/Europe 98 Technical Digest, 1998.

[11] J. Courtial, "Fractal multiple reduction imaging lasers," Opt. Commun. 174, pp. 235-241, 2000.

[12] J. Courtial, "Fractal laser mode structure due to iterated diffractive multiple imaging," J. Mod. Opt. 49, pp. 839-849, 2002.

[13] J. Courtial and M. J. Padgett, "Generation of self-reproducing fractal patterns using a multiple imaging system with feedback," J. Mod. Opt. 47, pp. 1469-1474, 2000.

[14] A. Forrester, J. Courtial, and M. J. Padgett, "Fractal generation using optical feedback with incoherent gain," Opt. Commun. 190, pp. 123-127, 2001.

[15] G. P. Karman and J. P. Woerdman, "Fractal structure of eigenmodes of unstable-cavity lasers," Opt. Lett. 23, pp. 1909-1911, 1998.

[16] G. P. Karman, G. S. McDonald, G. H. C. New, and J. P. Woerdman, "Fractal modes in unstable resonators," Nature 402, p. 138, 1999.

[17] M. Berry, "Fractal modes of unstable lasers with polygonal and circular mirrors," Opt. Commun. 200, pp. 321-330, 2001.

[18] M. Berry, C. Storm, and W. van Saarloos, "Theory of unstable laser modes: edge waves and fractality," Opt. Commun. 197, pp. 393-402, 2001.

[19] G. H. C. New, M. A. Yates, J. P. Woerdman, and G. S. McDonald, "Diffractive origin of fractal resonator modes," Opt. Commun. 193, pp. 261-266, 2001.

[20] M. A. Yates and G. H. C. New, "Fractal dimension of unstable resonator modes," Opt. Commun. 208, pp. 377-380, 2002.

[21] J. A. Loaiza, Experimental demonstration of the fractal nature of unstable-resonator modes. PhD thesis, Universiteit Leiden, 2005. 
[22] J. Courtial and M. J. Padgett, "Monitor-outside-a-monitor effect and self-similar fractal structure in the eigenmodes of unstable optical resonators," Phys. Rev. Lett. 85, pp. 5320-5323, 2000.

[23] C. M. G. Watterson, M. J. Padgett, and J. Courtial, "Classic-fractal eigenmodes of unstable canonical resonators," Opt. Commun. 223, pp. 17-23, 2003.

[24] J. Courtial, J. Leach, and M. J. Padgett, "Fractals in pixellated video feedback," Nature 414, p. 864, 2001.

[25] J. Leach, M. J. Padgett, and J. Courtial, "Fractals in pixellated video feedback," Contemp. Phys. 44, pp. 137-143, 2003.

[26] J. Nelson, J. Courtial, and G. Whyte, "Photorealistic visualization of imaging in canonical optical resonators," Am. J. Phys. 76(11), pp. 991-995, 2008.

[27] S. Leavey and J. Courtial, "Young TIM: A wave-optics simulator with slightly special powers." in preparation, 2014. 